\title{
Emotion regulation in times of COVID-19: A person-centered approach based on self-determination theory
}

\author{
Joachim Waterschoot ${ }^{1}$ (I) Sofie Morbée ${ }^{1} \cdot$ Branko Vermote $^{1} \cdot$ Katrijn Brenning $^{1} \cdot$ Nele Flamant $^{1}$. \\ Maarten Vansteenkiste ${ }^{1} \cdot$ Bart Soenens $^{1}$
}

Accepted: 9 December 2021

(C) The Author(s), under exclusive licence to Springer Science+Business Media, LLC, part of Springer Nature 2021

\begin{abstract}
Although the COVID-19 crisis is a worldwide threat to individuals' physical health and psychological well-being, not all people are equally susceptible to increased ill-being. One potentially important factor in individuals' vulnerability (versus resilience) to ill-being in the face of stress is emotion regulation. On the basis of Self-Determination Theory, this study examined the role of three emotion regulation styles in individuals' mental health during the COVID-19 crisis, that is, integration, suppression, and dysregulation. Participants were 6584 adults ( $77 \%$ female, $M_{\text {age }}=45.16$ years) who filled out well-validated measures of emotion regulation, depression, anxiety, life satisfaction, and sleep quality. To examine naturally occurring combinations of emotion regulation strategies, hierarchical k-means clustering was performed, yielding 3 profiles: (a) low scores on all strategies (indicating rather low overall levels of worry; 27\%), (b) high scores on integration only $(41 \%)$, and (c) high scores on suppression and dysregulation (32\%). Participants in the profiles scoring high on suppression and dysregulation displayed a less favorable pattern of outcomes (high ill-being, low life satisfaction, and poorer sleep quality) compared to the other two groups. Between-cluster differences remained significant even when taking into account the corona-related worries experienced by people. Overall, the findings underscore the important role of emotion regulation in individuals' mental health during mentally challenging periods such as the COVID-19 crisis. Practical implications and directions for future research are discussed.
\end{abstract}

Keywords COVID-19 $\cdot$ Emotion regulation $\cdot$ Self-determination theory $\cdot$ Mental health

The COVID-19 crisis brought about many different types of stressors that could undermine individuals' mental health (Vindegaard \& Benros, 2020). However, not all individuals are equally susceptible to this risk. Emotion regulation has been forwarded as a key factor to understand individual differences in the way people adjust to the COVID-19 crisis (Schimmenti et al., 2020). Unfortunately however, research on the role of emotion regulation on people's mental health during the COVID-19 crisis is scant. Furthermore, the few studies available (e.g., Jiang et al., 2020) typically focused on one or a few emotion regulation strategies, without considering the interplay between different emotion regulation strategies and the consequences of this interplay for mental

Joachim Waterschoot

Joachim.Waterschoot@ugent.be

1 Faculty of Psychology, Department of Developmental, Personality, and Social Psychology, Ghent University, Henri Dunantlaan 2, B-9000 Ghent, Belgium health in the context of COVID-19. Accordingly, there is a need for research identifying within-person combinations of different emotion regulation strategies (i.e., profiles) and examining associations between these profiles and individuals' mental health during the COVID-19 crisis.

Empirically, an examination of within-person combinations requires a person-centered statistical approach such as cluster analysis rather than a variable-centered approach (Bergman \& Wangby, 2014). Conceptually, such research needs to be conducted from a theoretical perspective distinguishing between multiple dimensions of emotion regulation that differ in terms of their functional role in mental health. The current study relies on Self-Determination Theory (SDT; Ryan \& Deci, 2017), a motivational theory offering a multidimensional conceptualization of emotion regulation and considering integrative regulation as the most effective type of emotion regulation.

The current study aims to contribute to the literature in two ways. First, at the empirical level, the study aims to strengthen the literature on COVID-19 and mental health 
by adopting a person-centered statistical approach to emotion regulation. Second, at the conceptual level, this study aims to be innovative by relying on SDT and by introducing the relatively new concept of integrative emotion regulation in research on COVID-19. Corresponding to these two anticipated contributions of the study, we next discuss (a) the importance of adopting a person-centered approach to emotion regulation during COVID-19 and (b) the value of considering integrative emotion regulation as a novel and potentially adaptive strategy to deal effectively with COVIDrelated stressors.

\section{Combinations of Emotion Regulation Strategies and Mental Health during COVID-19}

The COVID-19 crisis entails both immediate and more long-term stressors that potentially erode individuals' resources and mental health (Panchal et al., 2020). During the initial phases of the COVID-19 crisis, when governments across the world declared lockdown measures to contain the spreading of the virus, people had immediate concerns about their own health and about the availability of food and medical supplies (Carroll et al., 2020; Stephens et al., 2020). People also had more longterm concerns, for instance about their financial situation and about the duration and the unpredictability of the situation at large (Kämpfen et al., 2020). Confronted with these various stressors during the COVID-19 crisis, many people displayed increased risk for ill-being and decreased well-being. Indeed, several studies reported an elevated prevalence of depressive symptoms (Choi et al., 2020) and anxiety (Roy et al., 2020) as well as reductions in life satisfaction (Satici et al., 2020), sleep quality (Altena et al., 2020; Cellini et al., 2020), and overall well-being (Zhang et al., 2020). At the same time, people were found to differ widely in terms of adjustment to this challenging period, with many people also maintaining stable and high levels of mental health (e.g., Grossman et al., 2021; Wang et al., 2021).

Emotion regulation, which can be broadly defined as the process of 'monitoring, evaluation, and modification of emotional reactions' (Compas et al., 2017, p. 941), is key to understand individuals' responses to stress, worries, and concerns regarding negative life events (Parkinson et al., 2016). Therefore, there have been several calls to examine the role of emotion regulation in individuals' mental health during the COVID-19 period (Restubog et al., 2020; Schimmenti et al., 2020). However, to date, the number of studies examining emotion regulation in the context of COVID-19 is rather limited. The few studies currently available have focused mostly on the effects of cognitive reappraisal, that is, a mental re-interpretation of the situation to prevent a strong emotional response (Gross, 1998, 2014). To illustrate, Jiang et al. (2020) showed that Chinese adults who engaged in cognitive reappraisal were less likely to display symptoms of post-traumatic stress during the COVID-19 period. Similarly, Xu et al. (2020) found, among Chinese adults in quarantine, that cognitive reappraisal was related negatively to anxiety, and that this strategy also dampened the association between stress and anxiety. Further, cognitive re-appraisal was related negatively to COVID-19 worries and concerns (e.g., Luu, 2021; Prikhidko et al., 2020). For instance, Muñoz-Navarro et al. (2021) showed that the use of cognitive reappraisal resulted in less concerns regarding COVID-19 contamination, even when scoring high on general anxiety. Other studies have relied on general measures that aggregate several (supposedly) adaptive emotion regulation strategies (e.g., acceptance and cognitive reappraisal). Using such an aggregated measure, Jungmann and Witthöft (2020) found in a sample of German adults that adaptive emotion regulation related negatively to corona-related anxiety.

Although studies have begun to show that emotion regulation may play an important role in mental health during the COVID-19 crisis, research needs to go beyond a focus on one specific strategy or the use of summary measures of emotion regulation. That is, to better understand the role of emotion regulation in adaptation to the COVID19 crisis, it is important to rely on a multidimensional conceptualization distinguishing between emotion regulation strategies with differential consequences for mental health (e.g., Bergman \& Wangby, 2014). Further, because different emotion regulation strategies tend to co-occur within persons, these strategies should not be considered in isolation. People typically have different emotion regulation strategies available in their repertoire (Aldao et al., 2010; Blanke et al., 2020; Ford et al., 2019). As such, it is important to examine within-person profiles of emotion regulation strategies and associations of these profiles with mental health outcomes (Dixon-Gordon et al., 2015; van den Heuvel et al., 2020). Person-centered statistical analyses, such as cluster analysis, allow for the identification of such profiles, thereby detecting in a dataset the most common and naturally occurring combinations of emotion regulation strategies among all possible combinations of a given set of strategies. To conceptualize different emotion regulation strategies, this study relied on the theoretical framework of Self-Determination Theory (SDT; Ryan \& Deci, 2017), a general theory on motivation and mental health that provides a differentiated approach to emotion regulation. 


\section{A Self-Determination Theory Perspective on Emotion Regulation}

SDT distinguishes between three modes of emotion regulation that vary in their level of autonomy (Roth et al., 2009; Ryan et al., 2016). Integrative emotion regulation is the most autonomous type of regulation. It is characteristic of people who adopt a welcoming and accepting stance towards emotions, even when these emotions are painful and difficult (Roth et al., 2014; Shahar et al., 2018). People high on integrative emotion regulation take an active interest in their negative emotions, thereby trying to understand how these emotions inform them about their preferences and values. Ultimately, this better understanding of one's emotions also provides direction to people's actions. They know better how to act upon their emotions, feel free to either communicate or withhold their emotions, and see how they can respond more adequately to similar emotion-laden situations in the future (Benita, 2020; Roth et al., 2019). Emotional suppression represents a more controlled type of emotion regulation where people deny and minimize the strength and importance of emotions towards themselves (Kim et al., 2002; Roth et al., 2019). In addition to this experiential suppression, people also feel compelled to hide their negative emotions towards others, thereby suppressing the expression of emotions (Roth et al., 2009). With emotional dysregulation, people feel overwhelmed by their negative emotions, unable to understand the origins of these emotions, and incapable of canalizing or communicating about their emotions effectively (Houle \& Philippe, 2020). Therefore, they feel helpless in the face of negative emotions (Ryan et al., 2016).

The concept of integrative emotion regulation is relatively new (Roth et al., 2019). It is akin to, yet conceptually and empirically distinct from, related constructs such as acceptance (Dan-Glauser \& Gross, 2015) and mindfulness (Brown \& Ryan, 2003). Emotional integration shares with these constructs a welcoming and open attitude towards emotions. However, it also includes a more actionoriented attitude. It is not only about experiential openness for emotions but also about learning from these emotions and using emotions to inform future behaviors, decisions, and goals (Roth et al., 2019). Recent research has begun to corroborate the benefits associated with emotional integration, showing positive associations with personal wellbeing (Benita, 2020) and adaptive social outcomes such as prosocial behavior, empathy, and intimacy (Benita et al., 2017; Roth \& Assor, 2012). Longitudinal research showed that emotional integration even predicted increases across time in mental health (Brenning et al., 2015). Experimental studies demonstrated causal effects of situationally induced emotional integration on adaptive processing of threatening stimuli (Roth et al., 2014, 2018). In these experimental studies, people instructed to engage in emotional integration during a fear-eliciting movie, compared to participants instructed to suppress or minimize their emotions, displayed less anxiety and stress when confronted again with this movie on another occasion (Roth et al., 2014, 2018). In contrast, SDT-based studies (Benita, 2020; Brenning et al., 2015; Houle \& Philippe, 2020) and research in the broader literature on emotion regulation (Gross, 2015) have shown that both emotional suppression and dysregulation are related to lower mental health and greater risk for ill-being. These maladaptive effects of suppression and dysregulation have also been demonstrated in longitudinal (e.g., Brenning et al., 2015; McLaughlin et al., 2011) and experimental research (Gross, 1998; Gross \& Levenson, 1997).

Although research has begun to document the mental health benefits associated with emotional integration relative to suppression and dysregulation, no studies to date directly examined the relevance of these strategies for individuals' adjustment to the COVID-19 crisis. This is unfortunate because emotional integration is considered a resource for resilience in the context of highly stressful conditions (Roth et al., 2019; Weinstein et al., 2013). In contrast, suppression may have momentary benefits but is likely to backfire during more prolonged periods of stress, such as a stay-at-home lockdown (Gross, 2015). Similarly, dysregulation is a risk factor for mental health problems during unpredictable periods because it leads to a sense of uncontrollability (Compas et al., 2017).

Importantly, SDT's conceptualization of emotion regulation allows for an application of the person-oriented approach discussed before. Applied to the SDT taxonomy of emotion regulation, such a person-centered analysis could reveal a profile characterized by a combination of the two maladaptive emotion regulation strategies. It has indeed been argued that emotional suppression may go hand in hand with dysregulation across time (Gross, 2015). Because suppression is mentally effortful, people can suppress their negative emotions only for so long. During an extensive period of stress, emotional suppression may ultimately result in dysregulation once people's mental energy is drained. Another possibility is that some people combine both adaptive and more maladaptive emotion regulation strategies, with people for instance switching back and forth between emotional integration and dysregulation. Indeed, the openness to negative emotions characteristic of emotional integration may from time to time give rise to dysregulation among people who feel occasionally overwhelmed by their strong emotions. To the best of our knowledge, no studies to date adopted such a person-centered approach to the emotion regulation strategies identified in SDT. Such an approach can yield innovative findings that are important from both 
a fundamental and an applied perspective. In practice, people display combinations of emotion regulation strategies and practitioners (e.g., therapists and counselors) are more likely to recognize such profiles of strategies than isolated strategies.

\section{The Present Study}

Based on SDT, this study aimed to identify profiles of emotion regulation and to relate these profiles to individuals' mental health during a COVID-19 lockdown period. We focused on individuals' regulation of feelings of insecurity and threat as these emotions were very salient during the first weeks after the outbreak of the COVID-19 crisis. In general, we expected that individuals in profiles characterized by higher levels of emotional integration would display better mental health (i.e., more life satisfaction, better sleep, and less anxiety and depression) than individuals in profiles characterized by higher levels of suppression and dysregulation. Profiles characterized by a mix of adaptive and maladaptive strategies, if any, were expected to be situated in between profiles characterized uniquely by either adaptive or maladaptive strategies. In testing this hypothesis, we controlled for the degree to which people experienced worries due to the COVID-19 crisis. This is important because individuals in different profiles may be exposed to different levels of worries, with the amount of worry (rather than individuals' style of dealing with the worries) explaining differences between the profiles.

\section{Method}

\section{Procedure and Sample}

Data were collected during the first two weeks of the stayat-home lockdown in Belgium, specifically between March 19th and April 2nd, 2020. The questionnaire was presented online in Qualtrics and was completed by 6584 adults (77\% female) with a mean age of 45.16 years $(\mathrm{SD}=15.71$; range: 18-89). They all completed an active consent which stated their responses would be handled confidentially, that no negative consequences would follow after quitting the questionnaire, and that the data would be anonymized to avoid a link to their personal information. In this sample, $38 \%$ of the participants reported to be single or widowed and $62 \%$ was married or in a relationship (but not married). In terms of educational level, $72 \%$ graduated in higher education or university, $26 \%$ graduated in secondary school and only $2 \%$ did not finish secondary school. In response to a question about their current health (i.e., "Are you suffering from lung disease, diabetes, hypertension, autoimmune diseases?"), $81 \%$ of the participants reported having no medical problems at this moment. Finally, an open-ended item asked participants about their employment status. When participants indicated that they were currently employed, we also asked whether they work from home (or not). After coding participants' answers to this question, $47 \%$ indicated being employed and working from home, $24.3 \%$ indicated being employed and working on-site, $4.2 \%$ is unemployed, $3.8 \%$ is a student, $3.3 \%$ is disabled, $3.2 \%$ indicated being laid off, $2.1 \%$ is on sick leave and $12.1 \%$ is retired.

The survey was distributed online using the social networks of the researchers and multiple organizations and media (e.g., online newspapers). The instructions of the survey clarified that the focus of the study was on the psychological wellbeing of the Belgian population during the lockdown period. Both at the beginning and at the end of the questionnaire, contact information was provided in case participants needed psychological assistance or had questions regarding the study. Before participants were thanked, the possibility was provided to receive a summary of the results. On average, it took $11.35 \mathrm{~min}$ to complete the full questionnaire. The procedure used in this study was approved by the ethical committee of Ghent University (nr. 2020/37).

\section{Measures}

Worries Inspired by the measures of psychological (in) security used in Chen et al. (2015), four items were developed to assess people's COVID-specific worries during the lockdown. Following the stem "In the past week during the corona crisis, I was worried about...", participants were asked to indicate their COVID-specific worries concerning their health, financial situation, medication availability, and how the situation at large would evolve. Each item was rated on a scale ranging from 1 'not true at all' to 5 'totally true'. Internal consistency was moderate $(\alpha=.64)$.

Emotion Regulation To measure emotion regulation, we used the Dutch translation (Brenning et al., 2015) of the Emotion Regulation Inventory (Roth et al., 2009). Participants were asked to rate how they regulate feelings of threat and uncertainty related to the COVID-19 crisis during the previous week. For each subtype of emotion regulation, six items were rated on a scale ranging from 1 'not true at all' to 5 'totally true'. The scales for integrative emotion regulation (e.g., 'I examine my negative feelings to understand their sources', $\alpha=.78$ ), suppression (e.g., 'When I'm having these negative feelings, I make sure not to show them', $\alpha=.86$ ) and dysregulation (e.g., 'When I'm experiencing these negative feelings, I can't concentrate on other things I have to do', $\alpha=82$ ) displayed adequate internal consistency. 
Subjective Well-Being As for indicators of subjective wellbeing, participants rated single items tapping into their overall level of life satisfaction and sleep quality in the previous week (e.g., Fujita \& Diener, 2005). Using the most face valid item of the Satisfaction with Life Scale (Pavot \& Diener, 1993), participants were asked to rate to what extent they were satisfied with their life during the past week on a scale going from 1 (seldom or never, less than 1 day) to 4 (mostly or all the time, 5 to 7 days). Using the most face valid item from the Pittsburgh Sleep Quality Index (Buysse et al., 1989), participants were asked to rate their overall sleep in the past week on a scale ranging from 1 (very bad) to 4 (very good).

III-Being To measure participants' ill-being, we administered two scales tapping into anxiety and depressive symptoms experienced during the past week. Anxiety was measured with a 5-item version of the State Trait Anxiety Inventory (STAI, Marteau \& Bekker, 1992) and depressive symptoms were measured with a 6-item version (Van Hiel \& Vansteenkiste, 2009) of the Center for Epidemiological Studies - Depression scale (CES-D; Radloff, 1977). Items for both scales had to be rated on the same response scale, ranging from 1 (seldom or never, less than 1 day) to 4 (mostly or all the time, 5 to 7 days). Both questionnaires had acceptable reliability $\left(\alpha_{\text {anxiety }}=.87 ; \alpha_{\text {depression }}=.80\right)$.

\section{Plan of Analysis}

Preliminary Analysis Analyses were performed in R ( R Development Core Team, 2019). In a set of preliminary analyses, associations between background variables [gender, age, duration of the crisis (in weeks), educational level, health status, relationship status, employment status, and worries] and the study variables were examined with a Multivariate ANalysis of COVAriance (MANCOVA).

Clustering Procedure To perform person-centered analysis on the emotion regulation strategies, multivariate cluster analysis was used. Cluster analysis is ideally suited to determine which limited set of combinations of emotion regulation styles (among all theoretically possible combinations) naturally occur in a given sample. Much like a factor analysis reduces a set of items to a more limited number of underlying factors, cluster analysis aims to provide a parsimonious solution, thereby identifying the smallest possible number of profiles to represent the combinations of the study variables in the population. Specifically, we used Hierarchical K-Means clustering and we preferred this method to other commonly used person-oriented methods such as Latent Profile Analysis (LPA), for two reasons. First, we sought to identify clearly distinct and non-overlapping profiles of emotion regulation strategies. Because LPA assumes differences in the variances of the variables by profile, it allows for covariance between the profiles. By contrast, K-Means clustering does not include such geometric flexibility and as such results in profiles that do not overlap. Because Hierarchical K-Means clustering assumes 'statistical independence' between profiles, it also allows for a cleaner examination of between-group differences unaffected by statistical problems such as multicollinearity. As such, it allows for an easier interpretation. Second, LPA (which is based on the method of Gaussian Mixed Modelling) assumes multivariate normality within profiles, while Hierarchical K-Means clustering is model-free and a better fit with data that are not normally distributed within profiles.

The cluster analysis was performed in a number of steps. First, we standardized all study variables to make them comparable and to detect univariate outliers (based on a Median Absolute Deviation larger than 3, Leys et al., 2019) and multivariate outliers (i.e., values higher than a Median-based Mahalanobis distance of 22). Because the cluster analysis procedure is based on means, which are not robust to outliers, we decided to remove all detected outliers from the dataset (e.g., Hautamäki et al., 2005). Next, we performed a well-validated 2-step clustering procedure (Gore, 2000). It starts with a hierarchical clustering procedure (i.e., the most similar cases are linked to each other) from which the output is used as input for a K-Means clustering procedure (i.e., minimizing the within-cluster variation and maximizing the between-cluster variation). This 2-step approach has been proposed as the most efficient and valid way of clustering (Arai \& Barakbah, 2007). Instead of starting the K-Mean clustering algorithm with random starting points (i.e., minimizing the within-cluster variation by comparing each case to its position towards another cluster; Hartigan \& Wong, 1979), the centroids emerging from the Hierarchical clustering procedure were used as initial starting points to avoid a number of statistical issues (i.e., sensitivity to the order of cases, number of iterations, etc.). At the start, an Euclidian distance matrix is calculated, as the most common measure of 'cluster compactness', followed by the calculation of the agglomerative coefficient $(a c)$ for different linkage methods. The closer the $a c$ is to 1 , the more optimal the linkage method is for the dataset. In the second step, the K-Means clustering procedure is performed using the Hartigon and Wong algorithm (Hartigan \& Wong, 1979).

We evaluated the quality and the validity of the clustering procedure using three criteria. First, we checked the 'cluster tendency', which represents the suitability of the dataset to be clustered into meaningful clusters. To do so, we calculated the Hopkins statistic $H$ (Lawson \& Peter, 1990) which indicates better suitability when closer to 1 . Second, the optimal number of numbers is checked by four different validation techniques: the Elbow method (i.e. the number of clusters with both a minimum of within-cluster 
variation and a maximum of between-cluster variation), the Average Silhouette method (i.e. the number of clusters with the highest average silhouette, indicating the best quality of clustering; Kaufman \& Rousseeuw, 1990), the Gap statistic method (i.e. the number of clusters with the highest Gapstatistic; Tibshirani et al., 2001) and, at last, a summary of 30 indices reporting the most optimal number of clusters using the 'NbClust' function (Charrad et al., 2014), including the $\mathrm{CH}$ index (Calinski \& Harabasz, 1974). Third, the stability of the cluster solution was checked with a 'double split cross-validation' procedure (e.g., Vansteenkiste et al., 2009). Herein, the total sample is divided into two equal random samples on which the hierarchical clustering procedure is performed. Instead of using the results from this procedure as initial values for the K-Means clustering procedure, the centroids are switched between datasets. The stability is checked with a Cohen's Kappa-index $k$ testing the correspondence between the subsample-clustering results and the clustering results forming from the original clustering procedure. Acceptable cluster stability is assumed when $k$ is .60 or higher (Asendorpf et al., 2001). The final results of the clustering procedure will be presented in a barplot with the standardized cluster variables as a function of the cluster classification.

Between-Cluster Differences In the final step of the analyses, a MANCOVA is performed with the relevant covariates, cluster membership as a predictor, and with the dependent variables (subjective well-being and ill-being) as outcomes using Wilks' Lambda. In the univariate tests, post-hoc Tukey tests are performed for multiple comparison in case the predictor 'cluster' has more than 2 levels. Here, we applied the Bonferroni correction for $p$ values. The assumptions for linearity, normal residuals and homoscedasticity are checked.

\section{Results}

\section{Preliminary Analyses}

Pearson correlations and descriptive analyses can be found in Table 1. First, as a continuous demographic variable, age is related significantly to all study variables, with older participants reporting less dysregulation, integration, anxiety, depressive symptoms, and worries and reporting more suppression, higher life satisfaction, and better sleep quality. Corona-related worries are correlated positively with all three emotion regulation strategies, with the highest correlation for dysregulation and the lowest correlation with integration. Corona-related worries were also associated with more depressive symptoms and more anxiety and with poorer sleep quality and less life satisfaction.

As regards the emotion regulation strategies, dysregulation is related positively to both integration and suppression, with the latter two strategies being related negatively. Further, dysregulation and suppression both relate positively to more depressive and anxious symptoms and negatively to sleep quality and life satisfaction. Integration was largely unrelated to the dependent variables, demonstrating only very small correlations with more anxious and depressive symptoms and more life satisfaction.

Next, associations between categorical background variables (gender, educational level, health status, crisis duration, working status, and relationship status) and the study variables were inspected using a MANCOVA. Multivariate significant effects were found for all background variables. In terms of gender, women displayed more dysregulation and integration, more anxious symptoms, depressive symptoms, worries, and lower sleep quality compared to men (Wilks' $\lambda=.951 ; F(8,5926)=37.89, p<.001)$. Participants with a lower educational level showed higher scores for dysregulation, suppression and lower scores for integration and wellbeing (Wilks' $\lambda=.949 ; F(16,11,298)=18.83, p<.001)$.
Table 1 Means, standard deviations, and correlations between background and study variables

\begin{tabular}{lllllllllll}
\hline Variable & $\mathrm{M}$ & $\mathrm{SD}$ & 1 & 2 & 3 & 4 & 5 & 6 & 7 & 8 \\
\hline 1. Age & 45.26 & 15.66 & & & & & & & \\
2. Worries & 2.99 & .75 & $-.04^{* * *}$ & & & & & & & \\
3. Dysregulation & 2.27 & .72 & $-.20^{* * *}$ & $.38^{* * *}$ & & & & & & \\
4. Suppression & 2.31 & .74 & $.05^{* * *}$ & $.23^{* * *}$ & $.39^{* * * *}$ & & & & & \\
5. Integration & 3.28 & .66 & $-.08^{* * *}$ & $.07^{* * *}$ & $.11^{* * *}$ & $-.29^{* * *}$ & & & & \\
6. Anxiety & 2.20 & .80 & $-.20^{* * *}$ & $.56^{* * *}$ & $.60^{* * *}$ & $.32^{* * *}$ & $.06^{* * *}$ & & \\
7. Depression & 1.72 & .61 & $-.20^{* * * *}$ & $.45^{* * *}$ & $.61^{* * *}$ & $.37^{* * *}$ & .02 & $.77^{* * *}$ & & \\
8. Sleep quality & 2.83 & .73 & $.05^{* * * *}$ & $-.29^{* * *}$ & $-.34^{* * *}$ & $-.23^{* * *}$ & .00 & $-.44^{* * *}$ & $-.40^{* * *}$ & \\
9. Life satisfaction & 2.97 & .97 & $.14^{* * * *}$ & $-.31^{* * *}$ & $-.43^{* * *}$ & $-.31^{* * *}$ & $.07^{* * *}$ & $-.59^{* * *}$ & $-.58^{* * *}$ & $.30^{* * * *}$ \\
\hline
\end{tabular}

$M$ and $S D$ are used to represent mean and standard deviation, respectively. $* p<.05, * * p<.01, * * * p<$. 001 
Next, participants without health problems reported less suppressive emotion regulation, less worries and less depressive symptoms (Wilks' $\lambda=.975 ; F(16,11,850)=9.26, p<.001$ ). Participants who completed the questionnaire in the second week of the lockdown period reported lower integration, dysregulation, poorer sleep quality as well as more depressive symptoms compared to participants who completed the questionnaire in the first week (Wilks' $\lambda=.963 ; F(8$, $5926)=28.09, p<.001)$. In terms of employment status, participants working from home reported less suppressive emotion regulation, more integrative emotion regulation, better sleep quality, and fewer worries compared to those not working from home (Wilks' $\lambda=.881 ; F(59,31,799)=13.50$, $p<.001)$. Similarly, participants working from home had lower scores on these variables compared to all categories of unemployed participants. Only the retired status was an exception to this pattern, with those being retired reporting lower dysregulation, less depressive and anxiety symptoms, more life satisfaction, and similar sleep quality and worries compared to those working from home.

Finally, participants with a partner displayed lower integration, fewer symptoms of depression, and more dysregulation and life satisfaction than participants without a partner (Wilks' $\lambda=.873 ; F(40,25,816)=20.40, p<.001)$. Given these findings, we controlled for all of these covariates in the main analyses.

\section{A Person-Centered Approach: Hierarchical K-Means Analyses}

After standardization of the emotion regulation variables and inspection of the Mahalanobis distance values, $6 \%$ of the participants were identified as outliers and excluded from the cluster analysis (leaving a total $N$ of 6182 ). Studying the $a c$ 's for all linkage methods in the Hierarchical clustering procedure showed that the Ward's method was most optimal (.994) compared to the complete (.969), average (.925), and single (.798) linkage method.

To determine the number of clusters and the quality of the solution, the clustering procedure was explored for a range of 0 to 10 clusters. First, a $H$-statistic of .62 (>.50) was found, indicating a moderate clustering tendency. Figure 1 presents a graphical representation of all validation techniques to test the most optimal number of clusters in the current dataset. The elbow-method figure (Fig. 1, upper left) showed a balance between within- and between-cluster variance on the three-cluster solution. Next, two clusters have the highest silhouettes, followed by three and four clusters (Fig. 1, upper right). The Gap-statistic (Fig. 1, bottom left) indicates two and three clusters as the most optimal solutions. Finally, the frequency plot (Fig. 1, bottom right) shows a visualization of the majority rule, demonstrating that 9 out of 30 indices (including the $\mathrm{CH}$-index) point to the three-cluster solution
Fig. 1 Visualizations of cluster validation techniques
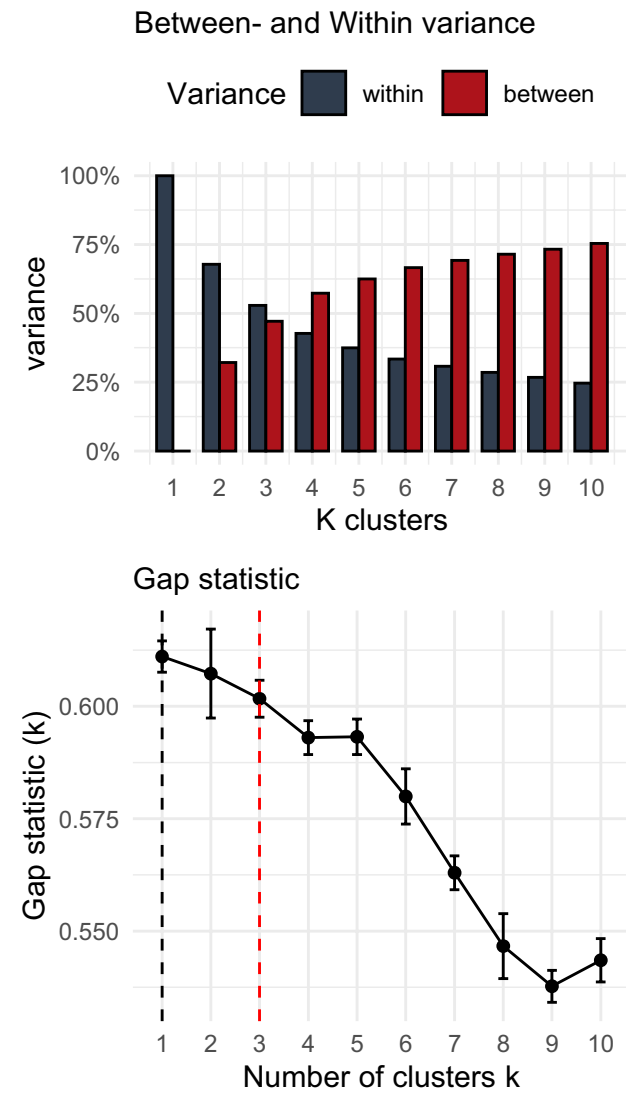

Average silhouette method

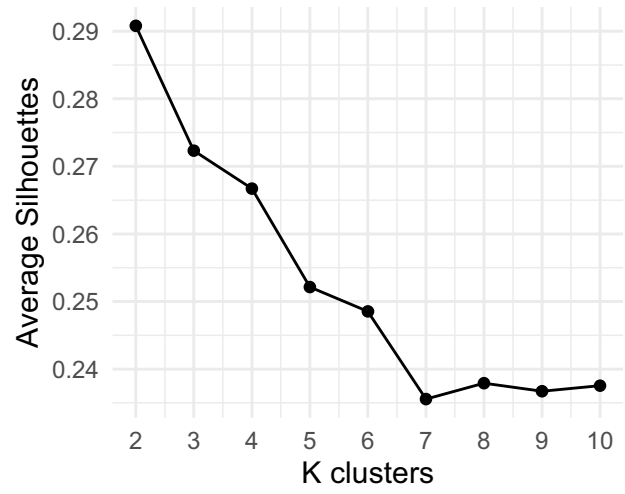

Summary frequency 30 indices

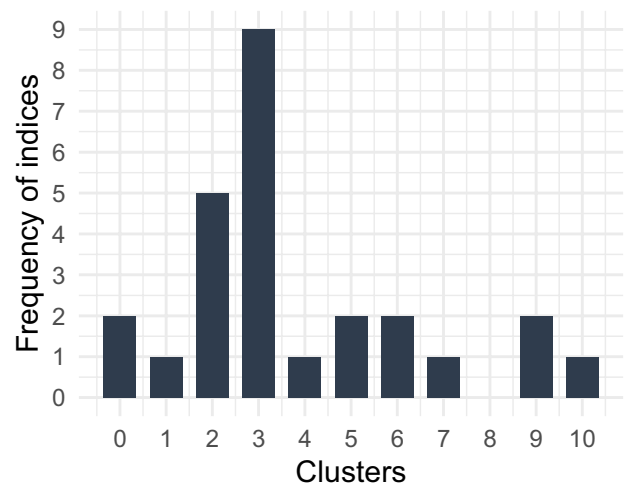


as the most optimal number of clusters. Considering all criteria, we chose the three-cluster solution as the most optimal representation of the current data.

The double-split cross-validation procedure to determine cluster stability revealed a weighted $k$ of $.60(z=47.46$, $\mathrm{p}<.001)$ for subset A and a weighted $k$ of $.48(\mathrm{z}=34.09$, $p<.001$ ) for subset B. The average of both kappa's is .54, indicating moderate cluster stability (Asendorpf et al., 2001).

The barplot in Fig. 2 shows the outcome of the clustering procedure, presenting the standardized values of the study variables per cluster. To test the differences between clusters in terms of the study variables, a MANCOVA with Tukey post-hoc tests was performed with dysregulation, integration, and suppression as dependent variables, cluster membership as a predictor, and all covariates included. A significant multivariate effect (Wilks' $\lambda=.206, F(6$, $\left.11788)=2362.91, p<.001, \eta^{2}=.55\right)$ was found. In terms of univariate differences, Cluster $1(27 \%)$ has the lowest scores for integration $(F(2,6190)=3166.77, p<.001$, $\left.\eta^{2}=.51\right)$ and dysregulation $(F(2,6190)=2679.75$, $p<.001, \eta^{2}=.46$ ). This cluster is characterized by low overall emotion regulation. Cluster $2(41 \%)$ has significantly higher scores on integration and the lowest scores on suppression $\left(F(2,6190)=2533.35, p<.001, \eta^{2}=.45\right)$. This cluster is characterized mainly by uniquely high values of integration. Cluster 3 (32\%) shows the highest scores for dysregulation and suppression. Because this cluster combines two non-autonomous emotion regulation strategies, it reflects overall dysfunctional emotion regulation.

To examine associations between cluster membership and participants' age and levels of worry, we conducted an ANOVA with cluster membership as a predictor. Participants in Cluster $1(M=2.74, S D=.68)$ showed the lowest levels of worry, followed by Cluster $2(M=2.92, S D=.70)$ and Cluster $3(M=3.29, S D=.70$; $F(1,5971)=265.68, p<.001)$, with differences between each of these clusters being significant. No effects were found for age $(F(1,5971)=3.38, p=.08)$. Next, a series of chi-squared tests demonstrated that male participants $\left(\chi^{2}(2)=133.15, p<.001\right)$ and participants working from home $\left(\chi^{2}(14)=123.24, p<.001\right)$ were overrepresented in Cluster 1, that participants with a higher education diploma $\left(\chi^{2}(4)=133.6, p<.001\right)$ and retired participants were overrepresented in Cluster 2, and that students and single participants $\left(\chi^{2}(2)=50.34, p<.001\right)$ were overrepresented in Cluster 3. Also, participants in Cluster 2 were more likely to have completed the questionnaire in week 1 of the crisis, compared to week $2\left(\chi^{2}(2)=24.17, p\right.$ $<$.001). No differences were found regarding health status $\left(\chi^{2}(4)=13.76, p<.001, p=.08\right)$.
Fig. 2 Barplot of clusters and features in terms of study variables

\section{Type of emotion regulation \\ Dysregulation $\square$ Suppression Integration}

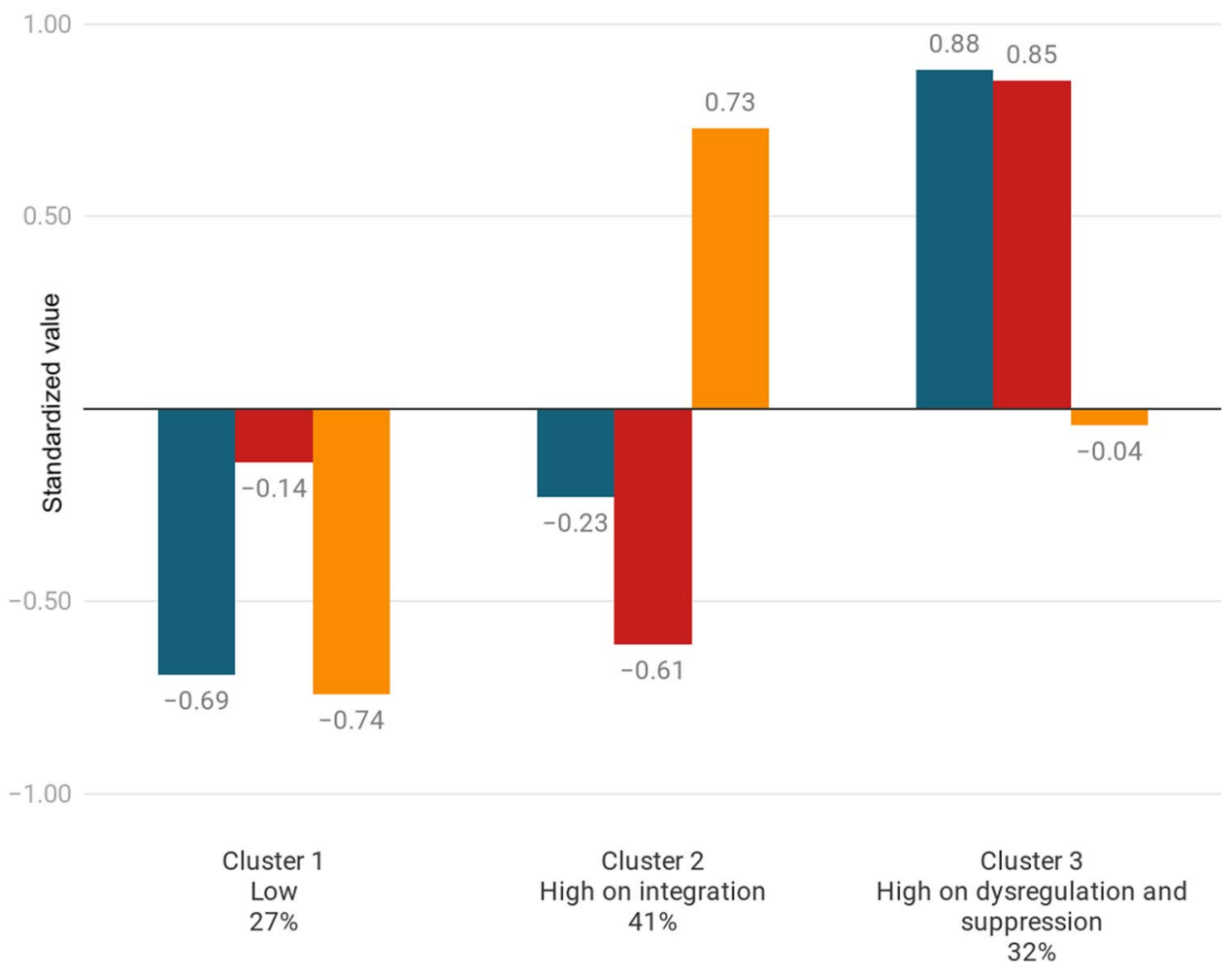


Table 2 Means and standard deviations per cluster with results of univariate tests

\begin{tabular}{|c|c|c|c|c|c|c|c|c|c|}
\hline & \multicolumn{2}{|c|}{$\begin{array}{l}\text { Cluster } 1 \text { (Low } \\
\text { overall emotion } \\
\text { regulation) }\end{array}$} & \multicolumn{2}{|c|}{$\begin{array}{l}\text { Cluster } 2 \text { (High } \\
\text { integration) }\end{array}$} & \multicolumn{2}{|c|}{$\begin{array}{l}\text { Cluster } 3 \text { (High } \\
\text { suppression } \\
\text { and dysregula- } \\
\text { tion) }\end{array}$} & \multirow[t]{2}{*}{$\mathrm{F}(2,5649)$} & \multirow[t]{2}{*}{$\mathrm{p}$-value } & \multirow[t]{2}{*}{$\eta^{2}$} \\
\hline & $M$ & $S D$ & $M$ & $S D$ & $M$ & $S D$ & & & \\
\hline 1. Anxiety & $1.77^{\mathrm{a}}$ & .61 & $2.05^{\mathrm{b}}$ & .70 & $2.71^{\mathrm{c}}$ & .73 & 482.31 & $<.001$ & .15 \\
\hline 2. Depression & $1.41^{\mathrm{a}}$ & .40 & $1.57^{\mathrm{b}}$ & .45 & $2.14^{\mathrm{c}}$ & .63 & 633.48 & $<.001$ & .18 \\
\hline 3. Sleep quality & $3.02^{\mathrm{c}}$ & .65 & $2.94^{\mathrm{b}}$ & .68 & $2.54^{\mathrm{a}}$ & .73 & 123.08 & $<.001$ & .04 \\
\hline 4. Life satisfaction & $3.27^{\mathrm{c}}$ & .85 & $3.19^{\mathrm{b}}$ & .86 & $2.45^{\mathrm{a}}$ & .96 & 283.88 & $<.001$ & .09 \\
\hline
\end{tabular}

Letters refer to annotation of Tukey post-hoc tests

\section{Associations between Cluster Membership and Mental Health}

To study between-cluster differences in terms of the mental health outcomes, accounting for the effect of coronarelated worries, a MANCOVA was conducted including all covariates (including worries) and cluster membership as a predictor of anxiety, depression, life satisfaction, and sleep quality. There was a multivariate significant effect of cluster membership (Wilks' $\lambda=.79 ; F(6,11292)=173.98, p<.001$, $\eta^{2}=.11$ ). The descriptive statistics with univariate tests and annotation of Tukey post-hoc tests are presented in Table 2. No assumptions were violated for any of the univariate analyses, the residuals being normally distributed, a diagonal Q-Q plot, and horizontal fitted values versus residual values plot with a random data cloud.

These results show that participants in Cluster 3 (i.e., high on dysregulation and suppression) show higher scores for ill-being (i.e., anxious and depressive symptoms) and lower scores for well-being (i.e., life satisfaction and sleep quality) compared to participants in the two other clusters. Participants in Cluster 1 (i.e., low overall emotion regulation) show the most adaptive pattern of outcomes with the highest scores for well-being and the lowest scores for ill-being. Participants in Cluster 2 (i.e., high on integration) scored in between those in Cluster 1 and 3 but leaned more closely to the adjustment profile of those in Cluster 1 than those in Cluster 3. However, we should be cautious about interpretations based on the $p$ values given the large sample size (i.e., increased type-I error). Therefore, the eta-squared (Table 2) provides a clearer understanding of the practical significance with small effects for sleep quality and life satisfaction and large effects for anxiety and depression (Cohen, 1988).

\section{Discussion}

This study aimed to investigate the role of three emotion regulation strategies, as defined in Self-Determination Theory, in individuals' mental health during the first two weeks of the first COVID-19 lockdown in Belgium. In doing this, we used a person-centered approach to identify within-person combinations of the emotion regulation strategies in relation to multiple measures of well- and ill-being.

\section{Identification of the Emotion Regulation Profiles}

This study identified three profiles of emotion regulation. Participants in the first profile overally displayed low emotion regulation, thereby scoring low on both adaptive and more maladaptive emotion regulation strategies. The few previous studies adopting a person-centered approach to emotion regulation (in pre-corona times) similarly identified a profile of 'low regulators' (Dixon-Gordon et al., 2015). Individuals in this profile likely experience few stressors and, as such, do not face high levels of distress that require regulation. We indeed found that participants in the low emotion regulation profile displayed the lowest levels of corona-related worries. With the current data, it cannot be determined whether these participants were confronted with less actual stressful events (e.g., less financial and health problems) or whether they are less inclined to subjectively experience such events as stressful. Future research could examine (e.g., using hypothetical scenarios) whether people in this profile have more benign and less catastrophizing appraisals of potentially stressful events than people in the other profiles.

A second profile was characterized by high levels of integrative emotion regulation and low levels of both dysregulation and suppression. From an SDT perspective, this profile can be viewed as adaptive because people in this profile display an open and sincere interest in their negative emotions, rather than minimizing and denying their emotions or feeling overwhelmed by their negative emotions (Roth et al., 2019). We did not find evidence for a profile characterized by both integrative emotion regulation and dysregulation. Although it seems plausible that some people who actively attend to negative emotions (i.e., integrative emotion regulation) risk evolving towards dysregulation, this combination of integrative 
emotion regulation and dysregulation was not observed in the current study. One element of integrative emotion regulation may be particularly helpful in avoiding the pitfalls of dysregulation, that is, individuals' use of negative emotions as input for behavior (Roth et al., 2019). People high on integrative emotion regulation not only attend to their emotions but also try to learn from these emotions and direct their behavior based on previous negative emotions. By doing so, they feel that negative emotions can be useful guideposts for behavioral adjustment. The latter experience probably protects against the sense of helplessness characteristic of dysregulation. Future research could adopt a person-centered analysis with different facets of integrative emotion regulation (thereby including more items for each facet than was the case in the current study). Possibly, such an analysis does yield a profile of people merely attending openly to their negative emotions (but failing to use these emotions in a constructive way as input for behavior) and at the same time feeling overwhelmed by these emotions, thus combining elements of integrative emotion regulation with dysregulation.

Participants in a third profile scored high on both emotional suppression and dysregulation and low on emotional integration. Because this profile combines a pressured and minimizing approach to negative emotions with helplessness and concerns of being overwhelmed with negative emotions, it can be considered a more maladaptive profile (e.g., Ciuluvica et al., 2019). Most likely, individuals in this profile attempt to dismiss and deny their negative emotions as long as possible. Because this downregulation of emotions is mentally draining, people at some point no longer manage to keep their negative emotions under control. These emotions then backfire with heightened valence, thereby exceeding the individual's capacity to regulate them effectively.

Although we had anticipated a profile characterized by emotional suppression only, strikingly we did not obtain evidence for this profile. Possibly, such a profile does exist in emotionally less troubling situations and during less taxing periods. Indeed, the fact that we found only evidence for a profile in which suppression co-occurs with dysregulation may be due to the unique historical period during which these data were collected. The COVID-19 lockdown period was challenging in many ways and stressful for many people. Under such challenging conditions, a unique reliance on emotional suppression may not be feasible across a long period of time and this strategy may inevitably spill over into dysregulation. Perhaps during less challenging and stressful periods, at least some people do manage to maintain high levels of emotion suppression without evolving towards dysregulation. Future research would do well to directly compare profiles of emotion regulation between challenging and more normative historical periods.
Associations of Profile Membership with Mental Health

Having identified these three emotion regulation profiles, a key aim of this study was to examine between-profile differences in terms of individuals' mental health during the COVID-19 lockdown. The most pronounced differences observed were between the maladaptive profile (combining suppression and dysregulation) and the two other profiles. Participants in the maladaptive profile reported the highest levels of anxiety and depression, the lowest levels of life satisfaction, and the poorest sleep quality. These findings are consistent with previous studies showing that both suppression and dysregulation increase the risk for mental health problems (Compare et al., 2014). The findings also confirm the SDT-based hypothesis that non-autonomous forms of emotion regulation, where people either feel compelled to dismiss their emotions or feel unable to regulate emotions effectively, render individuals more vulnerable to distress (Roth et al., 2019).

Differences between the profile characterized by low emotion regulation and the profile characterized predominantly by integrative emotion regulation were less pronounced than differences with the maladaptive emotion regulation profile. Participants in the low regulation profile even reported somewhat better adjustment than those in the integrative emotion regulation profile, thereby displaying less anxiety and depression and more life satisfaction and sleep quality. It should be noted that the effect size of these differences was small and that several of these effects reached significance only because of the large sample size. Still, the high levels of mental health displayed by people in the low regulation profile are interesting. The few person-centered studies identifying a similar profile of 'low regulators' also typically found that individuals in this profile are generally well-adjusted and do not differ substantially from individuals in an adaptive emotion regulation profile (Dixon-Gordon et al., 2015; van den Heuvel et al., 2020). Probably the main reason for these individuals' high levels of mental health is that they encounter fewer stressors than individuals in other profiles and, as such, do not feel a need to engage in emotion regulation. An important aim for future research is to examine how these individuals would respond when they suddenly do encounter negative life events (e.g., health problems due to COVID-19 or a worsening financial situation). Would most of these people still refrain from using emotion regulation strategies (perhaps relying only on problem-solving as a coping strategy) or would some of them transition to a profile characterized by higher levels of emotion regulation? In the latter case, what factors would determine whether they switch to a maladaptive emotion regulation profile or to a profile with more integrative emotion regulation? Longitudinal research would be ideally suited to examine the role 
of life events in transitions across time between emotion regulation profiles.

The finding that individuals in the integrative emotion regulation profile displayed higher levels of mental health compared to individuals in the maladaptive emotion regulation profile is consistent with SDT-based predictions and previous findings (e.g., Berking \& Wupperman, 2012). This finding indicates that, when people have a need to engage in emotion regulation, it is better for them to rely on integrative regulation than on the more maladaptive strategies. Possibly, the benefits associated with integrative emotion regulation manifest even stronger across time. Experimental studies have shown that integrative emotion regulation does not reduce anxiety immediately after having been exposed to a fear-eliciting stimulus but does protect against anxiety upon repeated exposure to this stimulus (Roth et al., 2014). Because we assessed integrative emotion regulation concurrently with the mental health outcomes, it is possible that the benefits of this emotion regulation style were somewhat underestimated and become even more visible across time. Thus, longitudinal research is needed to examine whether integrative emotion regulation predicts changes across time in mental health. Such research would do well to include also measures of negative life events. Perhaps integrative emotion regulation matters most when people are confronted with stress and adversity. Specifically, during challenging episodes, individuals in the integrative emotion regulation profile may fare better than individuals in the low regulation profile.

\section{Practical Implications}

Our findings have a number of potential implications for practice. First, because individuals combining suppression and dysregulation were found to display the highest levels of risk for mental health problems, they may benefit the most from interventions targeting emotion regulation. This is important because the effectiveness of universal prevention programs focusing on emotion regulation has been found to be relatively limited in terms of effect size (Durlak et al., 2011). One potential explanation for the limited effectiveness of universal prevention programs is that many participants following these programs, much like the individuals in the low emotion regulation profile identified in this study, do not encounter high levels of stress and negative affectivity. As such, these people may not experience a strong need to improve their emotion regulation skills. Other participants in these programs, such as those in the profile characterized by high emotional integration, may already routinely engage in effective emotion regulation and may also benefit only to a limited extent from the program. By using a selective prevention approach and by including mainly individuals with a vulnerable profile, such as those combining suppression and dysregulation, the cost-effectiveness of prevention programs could be enhanced.

Second, our findings point to the importance of targeting integrative emotion regulation in intervention programs or individual counseling. People struggling to deal effectively with stress and negative affect could be taught to approach negative emotions with open awareness and to consider such emotions as informational input for volitional action. Such interventions are particularly likely to strengthen individuals' resilience during challenging times as the COVID-19 crisis. Although, to the best of our knowledge, no intervention programs have been developed focusing specifically on emotional integration, findings from experimental studies are promising and show that people can be instructed to engage in emotional integration, with positive consequences for their affective functioning (Roth et al., 2014, 2018). As such, it seems worthwhile to complement existing emotion regulation programs, many of which focus on cognitive reappraisal and problem-solving, with information and exercises about emotional integration. Before engaging in a mental reappraisal of an emotional episode or actively trying to solve the problem causing the negative emotions, it may be important to learn people to first welcome and acknowledge their feelings, to understand the source of their own emotions, and then to decide volitionally how to act upon them (Roth et al., 2019).

\section{Limitations}

Although this study had a number of notable strengths (including the large sample, the inclusion of several emotion regulation strategies, and the person-centered approach), several limitations need to be addressed in future research. First, to collect the sample, we relied on a convenience sampling approach. Because participants were recruited through social media and because the assessment was online, the sample is not entirely representative for the population. A particular risk of this sampling approach is that people facing strong adversity during the COVID-19 crisis (e.g., people in very low SES conditions or confronted personally with severe health issues) were underrepresented in this study. This is unfortunate because contextual adversity threatens individuals' emotion regulation capacities. As such, future research would do well to actively recruit and oversample people living in at-risk conditions.

Second, due to the cross-sectional design of this study, we could not examine the effects of emotion regulation profiles on over-time changes in mental health. In addition, this design precludes conclusions about the direction of effects. Although we assumed, based on theorizing and previous longitudinal and experimental research (e.g., Brenning et al., 2015; Roth et al., 2014, 2018), that emotion regulation affects mental health, it seems equally likely that 
mental health problems contribute to more dysfunctional emotion regulation. People suffering from severe psychological distress may not have the mental energy available to engage in integrative emotion regulation and may instead look for shortcuts to minimize negative emotions as fast as possible (i.e., suppression). Longitudinal research measuring both emotion regulation and mental health repeatedly on several occasions is needed to examine the possibility of such transactional associations. Another relevant direction for future research in this regard is to experimentally induce the use of emotion regulation strategies. Research has shown that it is possible to induce integrative emotion regulation through experimental instructions. Using such instructions, people could be encouraged to engage in integrative emotion regulation on a daily basis during a period of time in the COVID-19 crisis. Their mental health could then be compared with a group of individuals receiving no such instructions. Experimental manipulations of emotion regulation allow for more causal conclusions and at the same time may inform interventions aimed at strengthening adaptive emotion regulation.

Third, because this study relied only on self-report measures some of the associations obtained may be inflated due to shared method bias. Future research would do well to adopt a multi-informant approach, with family members of the target individual for instance reporting on the individual's mental health. Another possibility is to include psychophysiological indicators of stress reactivity. For instance, experience sampling methodology would allow for the collection of self-report ratings and physiological indicators of stress several times during the day. Emotion regulation could then be examined as a moderator of the within-person association between (objectively assessed and subjectively experienced) stress and mental health problems. Moreover, the survey used in this study had to be short in order to motivate people to participate during a challenging time period. Therefore, the constructs were measured using a limited number of items and sometimes even with single items. Particularly with regard to the assessment of emotion regulation strategies, a disadvantage of this approach was that not all facets of these rich concepts could be measured. Accordingly, future research would do well to use more elaborate and multi-dimension measures (perhaps in a smaller sample with participants receiving an incentive for their more intensive efforts).

\section{Conclusion}

Using a person-centered approach and based on SDT as a theoretical framework, we found evidence for three profiles of emotion regulation during the COVID-19 crisis. One group of people overally displayed low emotion regulation, probably because they encountered few stressors and corresponding negative emotions. These people displayed the highest levels of mental health. Among the people who did engage in emotion regulation during the COVID-19 crisis, two qualitatively distinct profiles emerged. One profile involved high levels of emotional integration only and the other profile was characteristic of people who combined (and perhaps switched back forth between) suppression and dysregulation. People in the integrative emotion profile reported less distress, more life satisfaction, and better sleep quality than people engaging in the more maladaptive emotion regulation strategies. To the extent that future longitudinal and experimental research confirms the beneficial role of integrative emotion regulation in individuals' adaptation to the COVID-19 crisis (and to stress more generally), this emotion regulation strategy could be an important target for intervention.

Data Availability The datasets generated during and/or analyzed during the current study are available from the corresponding author on reasonable request.

\section{Declarations}

Conflict of Interest The authors declare they have no conflict of interest.

\section{References}

Aldao, A., Nolen-Hoeksema, S., \& Schweizer, S. (2010). Emotionregulation strategies across psychopathology: A meta-analytic review. Clinical Psychology Review, 30(2), 217-237. https://doi. org/10.1016/j.cpr.2009.11.004

Altena, E., Baglioni, C., Espie, C. A., Ellis, J., Gavriloff, D., Holzinger, B., Schlarb, A., Frase, L., Jernelöv, S., \& Riemann, D. (2020). Dealing with sleep problems during home confinement due to the COVID-19 outbreak: Practical recommendations from a task force of the European CBT-I academy. Journal of Sleep Research, 29(4), e13052. https://doi.org/10.1111/jsr.13052

Arai, K., \& Barakbah, A. R. (2007). Hierarchical K-means: An algorithm for centroids initialization for K-means. Reports of the Faculty of Science and Engineering, 36(1), 25-31.

Asendorpf, J. B., Borkenau, P., Ostendorf, F., \& van Aken, M. A. G. (2001). Carving personality description at its joints: Confirmation of three replicable personality prototypes for both children and adults. European Journal of Personality, 15, 169-198.

Benita, M. (2020). Freedom to feel: A self-determination theory account of emotion regulation. Social and Personality Psychology Compass, 14.

Benita, M., Levkovitz, T., \& Roth, G. (2017). Integrative emotion regulation predicts adolescents' prosocial behavior through the mediation of empathy. Learning and Instruction, 50, 14-20. https://doi. org/10.1016/j.learninstruc.2016.11.004

Bergman, L. R., \& Wangby, M. (2014). The person-oriented approach: A short theoretical and practical guide. Eesti Haridusteaduste Ajakiri.

Berking, M., \& Wupperman, P. (2012). Emotion regulation and mental health: Recent findings, current challenges, and future directions. 
Current Opinion in Psychiatry, 25(2), 128-134. https://doi.org/ 10.1097/YCO.0b013e3283503669

Blanke, E. S., Brose, A., Kalokerinos, E. K., Erbas, Y., Riediger, M., \& Kuppens, P. (2020). Mix it to fix it: Emotion regulation variability in daily life. Emotion, 20, 473-485.

Brenning, K., Soenens, B., Van Petegem, S., \& Vansteenkiste, M. (2015). Perceived maternal autonomy-support and early adolescent emotion regulation: A longitudinal study. Social Development, 24(3), 561-578.

Brown, K. W., \& Ryan, R. M. (2003). The benefits of being present: Mindfulness and its role in psychological well-being. Journal of Personality and Social Psychology, 84, 822-848.

Buysse, D. J., Reynolds 3rd, C. F., Monk, T. H., Berman, S. R., \& Kupfer, D. J. (1989). The Pittsburgh sleep quality index: A new instrument for psychiatric practice and research. Psychiatry Research, 28(2), 193-213. https://doi.org/10.1016/0165-1781(89)90047-4

Calinski, T., \& Harabasz, J. (1974). A dendrite method for cluster analysis communications in statistics. Theory and Methods, 3, 1-27.

Carroll, N., Sadowski, A., Laila, A., Hruska, V., Nixon, M., Ma, D., Haines, J., \& On Behalf Of The Guelph Family Health Study. (2020). The impact of COVID-19 on health behavior, stress, financial and food security among middle to high income Canadian families with young children. Nutrients, 12(8), 2352. https://doi. org/10.3390/nu12082352

Cellini, N., Canale, N., Mioni, G., \& Costa, S. (2020). Changes in sleep pattern, sense of time and digital media use during COVID-19 lockdown in Italy. Journal of Sleep Research, 29, e13074.

Charrad, M., Ghazzali, N., Boiteau, V., \& Niknafs, A. (2014). NbClust Package for Determining the Best Number of Clusters. R package version 2.0.3, URL http://CRAN.R-project.org/package=NbClust.

Chen, B., Vansteenkiste, M., Beyers, W., Boone, L., Deci, E. L., Duriez, B., Lens, W., Matos, L., Mouratidis, A., Ryan, R. M., Sheldon, K. M., Soenens, B., Van Petegem, S., Van der KaapDeeder, J., \& Verstuyf, J. (2015). Basic psychological need satisfaction, need frustration, and need strength across four cultures. Motivation and Emotion, 39, 216-236. https://doi.org/10.1007/ s11031-014-9450-1

Choi, E. P. H., Hui, B. P. H., \& Wan, E. Y. F. (2020). Depression and anxiety in Hong Kong during COVID-19. International Journal of Environmental Research and Public Health, 17(10), 3740. https:// doi.org/10.3390/ijerph17103740

Ciuluvica, C., Fulcheri, M., \& Amerio, P. (2019). Expressive suppression and negative affect, pathways of emotional dysregulation in psoriasis patients. Frontiers in Psychology, 10, 1907. https://doi. org/10.3389/fpsyg.2019.01907

Cohen, J. (1988). Statistical power analysis for the behavioral sciences (2nd edition). Hillsdale, New Jersey: L.

Compare, A., Zarbo, C., Shonin, E., Van Gordon, W., \& Marconi, C. (2014). Emotional Regulation and Depression: A Potential Mediator between Heart and Mind. Cardiovascular Psychiatry and Neurology, 2014, Article ID 324374, 10. https://doi.org/10. 1155/2014/324374.

Compas, B. E., Jaser, S. S., Bettis, A. H., Watson, K. H., Gruhn, M. A., Dunbar, J. P., Williams, E., \& Thigpen, J. C. (2017). Coping, emotion regulation, and psychopathology in childhood and adolescence: A meta-analysis and narrative review. Psychological Bulletin, 143(9), 939-991. https://doi.org/10.1037/bul0000110

Dan-Glauser, E. S., \& Gross, J. J. (2015). The temporal dynamics of emotional acceptance: Experience, expression, and physiology. Biological Psychology, 108, 1-12.

Dixon-Gordon, K. L., Aldao, A., \& De Los Reyes, A. (2015). Repertoires of emotion regulation: A person-centered approach to assessing emotion regulation strategies and links to psychopathology. Cognition and Emotion, 29, 1314-1325.

Durlak, J. A., Weissberg, R. P., Dymnicki, A. B., Taylor, R. D., \& Schellinger, K. B. (2011). The impact of enhancing students' social and emotional learning: A meta-analysis of school-based universal interventions. Child Development, 82(1), 405-432.

Ford, B. Q., Gross, J. J., \& Gruber, J. (2019). Broadening our field of view: The role of emotion regulation. Emotion Review, 11, 197-208.

Fujita, F., \& Diener, E. (2005). Life satisfaction set point: Stability and change. Journal of Personality and Social Psychology, 88(1), 158-164. https://doi.org/10.1037/0022-3514.88.1.158

Gore, P. (2000). Cluster analysis. In H. Tinsley \& S. Brown (Eds.), Handbook of applied multivariate statistics and mathematical modeling (pp. 297-321). Academic Press.

Gross, J. J. (1998). Antecedent-and response-focused emotion regulation: Divergent consequences for experience, expression, and physiology. Journal of Personality and Social Psychology, 74, 224-237. https://doi.org/10.1037/0022-3514.74.1.224

Gross, J. J. (2014). Emotion regulation: Conceptual and empirical foundations. In J. J. Gross (Ed.), Handbook of emotion regulation (pp. 3-20). Guilford Press.

Gross, J. J. (2015). Emotion regulation: Current status and future prospects. Psychological Inquiry, 26, 1-26.

Gross, J. J., \& Levenson, R. W. (1997). Hiding feelings: The acute effects of inhibiting negative and positive emotion. Journal of Abnormal Psychology, 106(1), 95-103. https://doi.org/10.1037/ 0021-843X.106.1.95

Grossman, E. S., Hoffman, Y. S., Palgi, Y., \& Shrira, A. (2021) COVID-19 related loneliness and sleep problems in older adults: Worries and resilience as potential moderators. Personality and Individual Differences, 168, 110371.

Hartigan, J. A., \& Wong, M. A. (1979). Algorithm AS 136: A k-means clustering algorithm. Applied Statistics, 28(1), 100-108.

Hautamäki, V., Cherednichenko, S., Kärkkäinen, I., Kinnunen, T., \& Fränti, P. (2005). Improving kmeans by outlier removal. In H. Kalviainen et al. (Eds.), SCIA 2005, LNCS (Vol. 3540, pp. 978-987).

Houle, I., \& Philippe, F. L. (2020). Is the negative always that bad? Or how emotion regulation and integration of negative memories can positively affect well-being. Journal of Personality, 1-13. https:// doi.org/10.1111/jopy.12544

Jiang, F., Deng, L., Zhang, L., Cai, Y., Cheung, C. W., \& Xia, Z. (2020). Review of the clinical characteristics of coronavirus disease 2019 (COVID-19). Journal of General Internal Medicine, 35, 1545-1549. https://doi.org/10.1007/s11606-020-05762-w

Jungmann, S. M., \& Witthöft, M. (2020). Health anxiety, cyberchondria, and coping in the current COVID-19 pandemic: Which factors are related to coronavirus anxiety? Journal of Anxiety Disorders, 73, 102239.

Kämpfen, F., Kohler, I. V., Ciancio, A., Bruine de Bruin, W., Maurer, J., \& Kohler, H. P. (2020). Predictors of mental health during the Covid-19 pandemic in the US: Role of economic concerns, health worries and social distancing. PLoS One, 15(11), e0241895.

Kaufman, L., \& Rousseeuw, P. J. (1990). Finding groups in data. John Wiley \& Sons.

Kim, Y., Deci, E. L., \& Zuckerman, M. (2002). The development of the self-regulation of withholding negative emotions questionnaire. Educational and Psychological Measurement, 62, 316-336.

Lawson, R. G., \& Peter, C. J. (1990). New index for clustering tendency and its application to chemical problems. Journal of Chemical Information and Computer Sciences, 30(1), 36-41.

Luu, T. T. (2021). Worker resilience during the COVID-19 crisis: The role of core beliefs challenge, emotion regulation, and family strain. Personality and Individual Differences, 179, 110784.

Leys, C., Delacre, M., Mora, Y. L., Lakens, D., \& Ley, C. (2019). How to classify, detect, and manage univariate and multivariate outliers, with emphasis on pre-registration. International Review of Social Psychology, 32(1). https://doi.org/10.5334/irsp.289

Marteau, T. M., \& Bekker, H. (1992). The development of a sixitem short-form of the state scale of the Spielberger state-trait 
anxiety inventory (STAI). The British Journal of Clinical Psychology, 31(3), 301-306. https://doi.org/10.1111/j.2044-8260. 1992.tb00997.x

McLaughlin, K. A., Hatzenbuehler, M. L., Mennin, D. S., \& NolenHoeksema, S. (2011). Emotion dysregulation and adolescent psychopathology: A prospective study. Behaviour Research and Therapy, 49(9), 544-554. https://doi.org/10.1016/j.brat.2011. 06.003

Muñoz-Navarro, R., Malonda, E., Llorca-Mestre, A., Cano-Vindel, A., \& Fernández-Berrocal, P. (2021). Worry about COVID19 contagion and general anxiety: Moderation and mediation effects of cognitive emotion regulation. Journal of Psychiatric Research, 137, 311-318.

Panchal, N., Kamal, R., Orgera, K., Cox, C., Garfield, R., Hamel, L., Muñana, C., \& Chidambaram, P. (2020). The implications of COVID-19 for mental health and substance use. Kaiser Family Foundation (KFF). Retrieved from: www.kff.org/coronaviruscovid-19/issue-brief/the-implications-of-covid-19-for-mentalhealth-and-substance-use/

Parkinson, B., Simons, G., \& Niven, K. (2016). Sharing concerns: Interpersonal worry regulation in romantic couples. Emotion, 16(4), 449

Pavot, W., \& Diener, E. (1993). Review of the satisfaction with life scale. Psychological Assessment, 5(2), 164-172. https://doi.org/ 10.1037/1040-3590.5.2.164

Prikhidko, A., Long, H., \& Wheaton, M. G. (2020). The effect of concerns about COVID-19 on anxiety, stress, parental burnout, and emotion regulation: The role of susceptibility to digital emotion contagion. Frontiers in Public Health, 8, 894.

R Development Core Team. (2019). R: A language and environment for statistical computing. R Foundation for Statistical Computing.

Radloff, L. S. (1977). The CES-D scale: A self-report depression scale for research in the general population. Retrieved from the University of Minnesota Digital Conservancy, https://hdl.handle.net/11299/98561.

Restubog, S., Ocampo, A., \& Wang, L. (2020). Taking control amidst the chaos: Emotion regulation during the COVID-19 pandemic. Journal of Vocational Behavior, 119, 103440. https://doi.org/ 10.1016/j.jvb.2020.103440

Roth, G., \& Assor, A. (2012). The cost of parental pressure to express emotions: Conditional regard and autonomy support as predictors of emotion regulation and intimacy. Journal of Adolescence, 35, 799-808. https://doi.org/10.1016/j.adolescence. 2011.11.005

Roth, G., Assor, A., Niemiec, C. P., Ryan, R. M., \& Deci, E. L. (2009). The emotional and academic consequences of parental conditional regard: Comparing conditional positive regard, conditional negative regard, and autonomy support as parenting practices. Developmental Psychology, 45, 1119-1142.

Roth, G., Benita, M., Amrani, C., Shachar, B.-H., Asoulin, H., Moed, A., Bibi, U., \& Kanat-Maymon, Y. (2014). Integration of negative emotional experience versus suppression: Addressing the question of adaptive functioning. Emotion, 14(5), 908-919. https://doi.org/10.1037/a0037051

Roth, G., Shahar, B.-H., Zohar-Shefer, Y., Benita, M., Moed, A., Bibi, U., Kanat-Maymon, Y., \& Ryan, R. M. (2018). Benefits of emotional integration and costs of emotional distancing. Journal of Personality, 86, 919-934. https://doi.org/10.1111/ jopy. 12366

Roth, G., Vansteenkiste, M., \& Ryan, R. (2019). Integrative emotion regulation: Process and development from a self-determination theory perspective. Development and Psychopathology, 31(3), 945-956. https://doi.org/10.1017/S0954579419000403
Roy, D., Sarvodaya, T., Sujita, K., Nivedita, S., Sudhir, K., \& Vika, K. (2020). Study of knowledge, attitude, anxiety \& perceived mental healthcare need in Indian population during COVID-19 pandemic. Asian Journal of Psychiatry, 51, 102083. https://doi. org/10.1016/j.ajp.2020.102083

Ryan, R. M., \& Deci, E. L. (2017). Self-determination theory: Basic psychological needs in motivation, development, and wellness. The Guilford Press. https://doi.org/10.1521/978.14625/28806

Ryan, R. M., Deci, E. L., \& Vansteenkiste, M. (2016). Autonomy and autonomy disturbances in self-development and psychopathology: Research on motivation, attachment, and clinical process. In D. Cicchetti (Ed.), Developmental psychopathology: Theory and method (pp. 385-438). John Wiley \& Sons. https://doi.org/ 10.1002/9781119125556.devpsy109

Satici, B., Gocet-Tekin, E., Deniz, M. E., \& Satici, S. A. (2020). Adaptation of the fear of COVID-19 scale: Its association with psychological distress and life satisfaction in Turkey. International Journal of Mental Health and Addiction, Epub ahead of print. https://doi.org/10.1007/s11469-020-00294-0.

Schimmenti, A., Billieux, J., \& Starcevic, V. (2020). The four horsemen of fear: An integrated model of understanding fear experiences during the COVID-19 pandemic. Clinical Neuropsychiatry, 17, 41-45.

Shahar, B. H., Kalman-Halevi, M., \& Roth, G. (2018). Emotion regulation and intimacy quality: The consequences of emotional integration, emotional distancing, and suppression. Journal of Social and Personal Relationships. Advance online publication. https://doi.org/10.1177/0265407518816881.

Stephens, E. C., Martin, G., van Wijk, M., Timsina, J., \& Snowe, V. (2020). Impacts of COVID19 on agricultural and food systems worldwide and on progress to the sustainable development goals. Agricultural System, 183, 102873. https://doi.org/ 10.1016/j.agsy.2020.102873

Tibshirani, R., Walther, G., \& Hastie, T. (2001). Estimating the number of clusters in a data set via the gap statistic. Journal of the Royal Statistical Society: Series B (Statistical Methodology), 63(2), 411-423.

van den Heuvel, M. W., Stikkelbroek, Y. A., Bodden, D. H., \& van Baar, A. L. (2020). Coping with stressful life events: Cognitive emotion regulation profiles and depressive symptoms in adolescents. Development and Psychopathology, 32, 985-995.

Van Hiel, A., \& Vansteenkiste, M. (2009). Ambitions fullfilled? The effects of intrinsic and extrinsic goal attainment on older adults' ego-integrity and death attitudes. International Journal of Aging \& Human Development, 68(1), 27-51.

Vansteenkiste, M., Ryan, R. M., \& Soenens, B. (2020). Basic psychological need theory: Advancements, critical themes, and future directions. Motivation and Emotion, 44, 1-31. https://doi.org/ 10.1007/s11031-019-09818-1

Vansteenkiste, M., Sierens, E., Soenens, B., Luyckx, K., \& Lens, W. (2009). Motivational profiles from a self-determination perspective: The quality of motivation matters. Journal of Educational Psychology, 101, 671-688.

Vindegaard, N., \& Benros, M. E. (2020). COVID-19 pandemic and mental health consequences: Systematic review of the current evidence. Brain, Behavior, and Immunity, 89, 531-542.

Wang, Q.-Q., Fang, Y.-Y., Huang, H.-L., Lv, W.-J., Wang, X.-X., Yang, T.-T., Yuan, J.-M., Gao, Y., Qian, R.-L., \& Zhang, Y.-H. (2021). Anxiety, depression and cognitive emotion regulation strategies in Chinese nurses during the COVID-19 outbreak. Journal of Nursing Management.

Weinstein, N., Przybylski, A. K., \& Ryan, R. M. (2013). The integrative process: New research and future directions. Current Directions in Psychological Science, 22, 69-74. 
Xu, C., Xu, Y., Xu, S., Zhang, Q., Liu, X., Shao, Y., Xu, X., Peng, L., \& Li, M. (2020). Cognitive reappraisal and the association between perceived stress and anxiety symptoms in COVID-19 isolated people. Frontiers in Psychiatry, 11, 858. https://doi. org/10.3389/fpsyt.2020.00858
Zhang, X., Wang, Y., Lyu, H., Zhang, Y., Liu, Y., \& Luo, J. (2020). The influence of COVID-19 on well-being. https://doi.org/10. 31234/osf.io/znj7h

Publisher's Note Springer Nature remains neutral with regard to jurisdictional claims in published maps and institutional affiliations. 